\title{
UAVs as Mobile Infrastructure: Addressing Battery Lifetime
}

\author{
Boris Galkin, Jacek Kibiłda, and Luiz A. DaSilva \\ CONNECT, Trinity College Dublin, Ireland \\ E-mail: \{galkinb,kibildj,dasilval\}@tcd.ie
}

\begin{abstract}
Unmanned Aerial Vehicles (UAVs) can play an important role in next generation cellular networks, acting as flying infrastructure which can serve ground users when regular infrastructure is overloaded or unavailable. As these devices operate wirelessly they rely on an internal battery for their power supply, which limits the amount of time they can operate over an area of interest before having to recharge. To accommodate this limitation UAV networks will have to rely on dedicated infrastructure to recharge the UAVs in-between deployments. In this article, we outline three battery charging options that may be considered by a network operator and use simulations to demonstrate the performance impact of incorporating those options into a cellular network where UAV infrastructure provides wireless service.
\end{abstract}

Index Terms-UAV networks, coverage probability, battery lifetime, wireless power transfer

\section{INTRODUCTION}

In recent years remote-controlled flying devices (UAVs) have expanded from the domain of military applications into civilian markets, with millions of consumer-grade UAVs being sold every year around the world. This evolution is attributed to a number of recent technological developments which have made it possible to develop small, affordable UAVs capable of carrying out a variety of tasks using on-board devices. These tasks include the use of UAVs in emergency applications, for industrial and agricultural inspections, and for package delivery. There is a growing interest among the wireless research community in the possibility of using UAVs as flying infrastructure acting alongside, or in place of, terrestrial networks, in a variety of scenarios.

UAV-mounted communications infrastructure is a complete paradigm shift which can bring several key benefits over the existing mobile network infrastructure, including:

1) UAVs, due to their airborne nature, can establish much higher quality channels to a terrestrial receiver, with significantly lower signal attenuation. Whereas a macro base station mounted on a building rooftop will experience non-Line-of-Sight (NLOS) propagation conditions to its associated ground users due to the buildings in the way, the UAV can adjust its height to hover high above such obstacles. This benefit is particularly significant in urban areas with high building density.

2) The UAVs, as they can move on command, can optimise their locations in real-time with respect to the location of the traffic demand, and can then readjust as the demand changes. This is in stark contrast to existing infrastructure, which is fixed in place and relies on careful site planning on the part of the network operators to ensure efficient service. By optimising their locations in real time the UAV infrastructure can achieve greater service efficiency, while also reducing overheads.

While they introduce a variety of benefits to wireless networks the UAVs are limited by their on-board battery life, which restricts the length of time that a given UAV can stay in the air. As a consequence of this the UAV-mounted infrastructure can only provide temporary service to an area of interest, unless a solution is implemented to address the battery life issue.

There has been a number of publications from the wireless community on the topic of optimising the energy efficiency of a UAV with respect to the service it delivers to its users. In [1] the authors consider an Internet of Things (IoT) framework where a centralised network controller entity selects UAVs from a fleet of available devices, based on the specific requirements of the task and also the energy consumption requirements. The authors set up an optimisation problem based on either minimising the energy consumption or the task completion delay, and demonstrate via simulations the resulting performance.

The work in [2] considers aggregating multiple UAVs into a single cluster, with the clusterhead UAV routing data from the other UAVs to the ground station. Using a fuzzy logic algorithm the authors demonstrate how the UAVs can be aggregated into clusters, with a clusterhead selected according to the cluster centroid. The authors then use simulations to demonstrate that this approach can reduce UAV energy expenditure.

The authors of [3] propose a path planning algorithm to determine which UAV to send to a given set of service locations, to minimise the total energy consumed by the UAVs for travel. The authors propose solving the problem using mixed integer linear programming; for comparison the authors also propose a greedy algorithm and an algorithm which minimises UAV path overlap. Using simulations and energy consumption values obtained from an off-the-shelf consumer UAV the authors demonstrate the reduction in UAV network energy consumption that can be obtained using their algorithm.

In [4] the authors investigate the possibility of landing UAVs on rooftops during operation to reduce their power consumption and thereby increase their operating time. The authors consider a scenario where a UAV has to provide 
service to a number of users and there are several available landing sites on rooftops in the area; the authors optimise the trajectory of the UAV and its velocity to maximise total achievable data rate over a time period of interest.

The reader may note from the above overview of the stateof-the-art that the wireless community tends to consider the battery life issue from the perspective of optimising UAV behaviour, while not considering the impact of the charging infrastructure itself. UAVs are highly flexible devices whose mobility can be leveraged to maximise their performance; however, optimising the UAV mobility is not sufficient for creating a viable UAV network. Network operators must design the UAV network from the ground up to accomodate UAV recharging between deployments, which includes not just designing the UAVs themselves, but also the infrastructure for charging them. There are a variety of ways that ground charging infrastructure can be deployed in an area of interest to support the UAV network: how these deployments will affect the performance of the UAV network and ultimately the service experienced by the users needs to be carefully analysed.

In this article we explore several approaches for a network operator to address the UAV battery lifetime issue and design a UAV network that enables continuous wireless coverage. We evaluate the sort of network performance that can be achieved by implementing various types of charging infrastructure, and we discuss the relative strengths and weaknesses of each approach. Furthermore, we provide a high level overview of the developments being made in the field of battery technology, and demonstrate how they may improve UAV performance in the foreseeable future.

\section{UAV CHARACTERISTICS}

We begin by providing a short discussion on the type of UAVs currently available on the civilian market and which variants we expect to be used for flying infrastructure. UAVs vary greatly in size, with the smallest UAVs weighing less than $1 \mathrm{~kg}$ and fitting comfortably inside personal bags, while the larger UAVs are the size of manned aircraft. The variance in size also corresponds to different regulations and restrictions. The Federal Aviation Administration (FAA) and the European Aviation Safety Agency (EASA) currently restrict UAVs with a take-off weight below $25 \mathrm{~kg}$ to operate at heights below $120 \mathrm{~m}$, which corresponds to unregulated airspace which manned aircraft do not operate in, whereas the larger UAVs are required to use regulated airspace and coordinate with air traffic control. Companies such as Google and Facebook have expressed interest in using large, higher-altitude UAVs for providing basic wireless connectivity to remote areas with limited existing infrastructure. For dense, urban areas the small, low altitude UAVs are more appropriate, as they are safer to use due to their small size and they can fine-tune their positioning in $3 \mathrm{D}$ space in a manner that is unavailable to the larger aircraft. Note that accurate positioning of the UAVs in 3D space may prove challenging in an urban environment, particularly under windy conditions. As accurate positioning and resilience against weather effects are a prerequisite for safe operation in urban areas, we expect UAV platforms to carry dedicated hardware which enables them to accurately adjust their coordinates.

Low altitude UAV designs can be separated into two categories based on their method of flight. The first category of UAVs is referred to as fixed-wing, and corresponds to an airplane design, where UAVs have wings which generate lift from air passing underneath. The second category is the rotorwing, where the UAVs have several rotors with propellors which push air downwards and generate enough thrust to counter the force of gravity on the UAV. Both designs have advantages and disadvantages. The advantage of the fixedwing design is that it allows the UAV to fly with less thrust from its motors due to the behaviour of aerodynamic flow. Less thrust needed to fly corresponds directly to less energy consumed, which means a longer flight time for a given battery. The disadvantage of this design is that the UAV must always be moving forward at a certain minimum velocity to generate enough thrust to stay in the air, and as a consequence it is impossible to keep the UAV hovering above a certain location of interest. It also means that the UAV requires a large open area for take-off and landing, and in a dense urban environment open areas of suitable size may not be available. Because of this, we consider the rotor-wing UAV design to be the most appropriate for operating in an urban environment.

In our previous work [5] we have explored the performance of a low altitude, rotor-wing UAV network operating above user hotspots. We demonstrated that UAVs can leverage their height to find a performance sweet-spot which balances their ability to deliver a wireless signal to a typical user while also minimising the amount of interference the user experiences. The height which gives this optimum performance is a function of the density of the UAVs, their antenna configuration, the Line-of-Sight (LOS) blocking buildings in the environment, and also the size of the user hotspots themselves. We expect that an intelligent UAV network will position UAVs at the heights which give the best performance: the consequence of this is that the UAVs will have to expend a certain amount of their total battery power on getting into position to serve the users, with the exact amount of battery power (and the resulting battery life left) being highly dependent on the environmental parameters.

In the following sections we consider the same UAV small cell use case scenario as in [5], with the UAVs hovering at optimum heights above user hotspots. The network and environmental parameters are taken from [5]. We consider a charging station density of $1 / \mathrm{km}^{2}$ and a user hotspot density of $5 / \mathrm{km}^{2}$. The charging stations and the hotspots are positioned independently of each other, to reflect the fact that in real-world scenarios the locations of the hotspots may not be known in advance, and suitable locations for the charging stations may be limited. UAV power consumption parameters are taken from [6], with a battery weight of $0.4 \mathrm{Kg}$.

\section{UAV SWAPPING}

One of the most straightforward ways of building a UAV network around the limited battery life of the UAV is to 


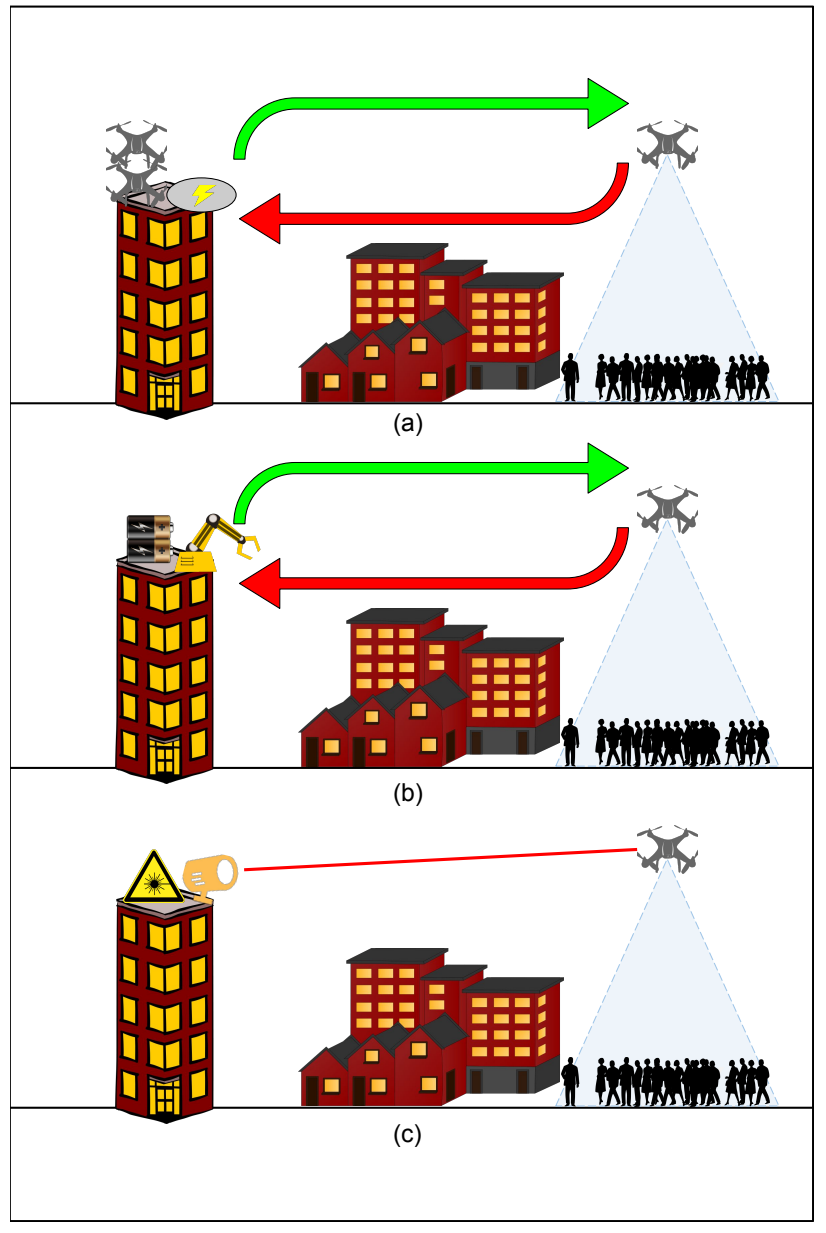

Fig. 1. Proposed UAV battery management solutions: (a) cycling through multiple UAVs to cover a hotspot; (b) hotswapping batteries of a single UAV covering a hotspot; (c) using lasers to wirelessly power a UAV.

sequentially switch out low-power UAVs with ones that are fully charged, as depicted in Fig. 1(a). In this scenario, for each UAV that is operating above a user hotspot there are several other UAVs being charged at a charging station, waiting to be deployed. When the first UAV must return to its charging station to recharge it will be replaced by a second UAV, which in turn will be replaced by a third, and so on, until the first UAV is fully charged at the charging station and is ready to be deployed again. By having a sufficiently large number of backup UAVs and by timing their deployments such that one UAV hands over its hotspot seamlessly to another UAV the network can provide continuous, uninterrupted service to an area.

The number of backup UAVs that must be kept in a state of readiness for a given hotspot will be determined by UAV "downtime", that is, the length of time the UAV will need to travel back to its charging station, recharge, and return to the hotspot. The longer the recharge time, the more UAVs are needed to substitute it before it can deploy again. In Fig. 2 we demonstrate the number of backup UAVs that are needed, on average, for a given operating UAV as a function of the UAV recharging power and the speed that the UAVs move at. If we consider commercially-available UAVs today to have a charging power of $180 \mathrm{~W}$ we may require as little

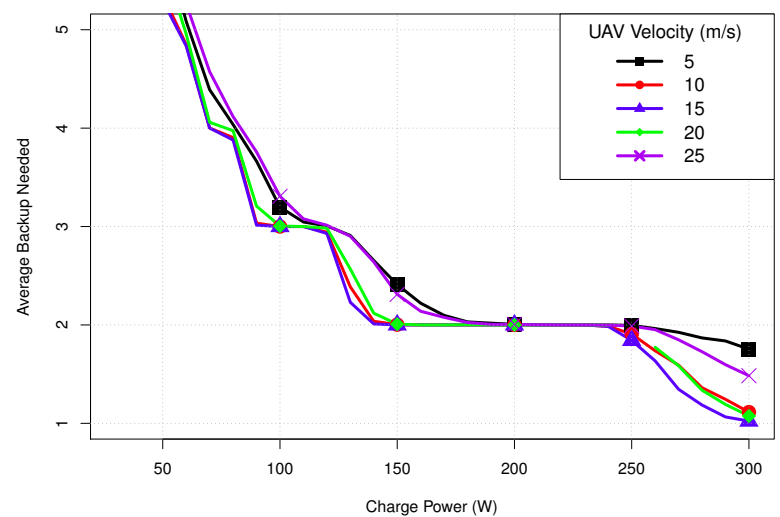

Fig. 2. Average number of UAV backups that are needed for each UAV, as a function of UAV speed and the charger power.

as two backup UAVs for each operating UAV in the network to ensure continuous coverage. The figure clearly shows that developing new battery charging technologies to enable faster energy transfer and reduce charging time is needed to reduce the number of backup UAVs and make the UAV network more affordable. Note that the UAV horizontal velocity does not appear to have a significant impact on the number of backups: a higher velocity allows UAVs to spend less time on travel; however, it also consumes more battery power [6].

\section{BATTERY HOTSWAPPING}

The majority of high-end UAVs nowadays are designed with external battery packs that can be detached from the UAV, thus enabling fast swapping of batteries by the UAV operator. Certain high-end models even carry two external batteries, both for safety reasons and to enable battery hotswapping. Battery hotswapping is when a UAV battery is replaced without the UAV being powered off, which allows it to return to its regular operation the moment the new battery is in place. The drawback of battery hotswapping is that it currently requires a human operator to carry out the mechanical operation of detaching the depleted battery and inserting a new one into the UAV. This introduces human labour into what may otherwise be an automated network. To address this, researchers have explored the concept of automated battery swapping stations, where robotic actuators are used to switch out batteries. The authors of [7] demonstrate a working prototype of such a station, showing how a UAV can automatically land into the charging station and have its battery swapped out within 60 seconds.

To demonstrate the benefits of this setup we consider the scenario depicted in Fig. 1(b). A UAV provides service above a user hotspot until its battery is depleted and it must return to its charging station. There, its battery is hotswapped with a backup battery and it returns to its hotspot, while its previous battery is charged up. Instead of having several backup UAVs we have several backup batteries, which reduces the cost of the infrastructure; however, because we only have one UAV per hotspot the hotspot will not be serviced for the length of time it takes the UAV to move to its charging station, hotswap its battery, and return back. Fig. 3 shows the duration 


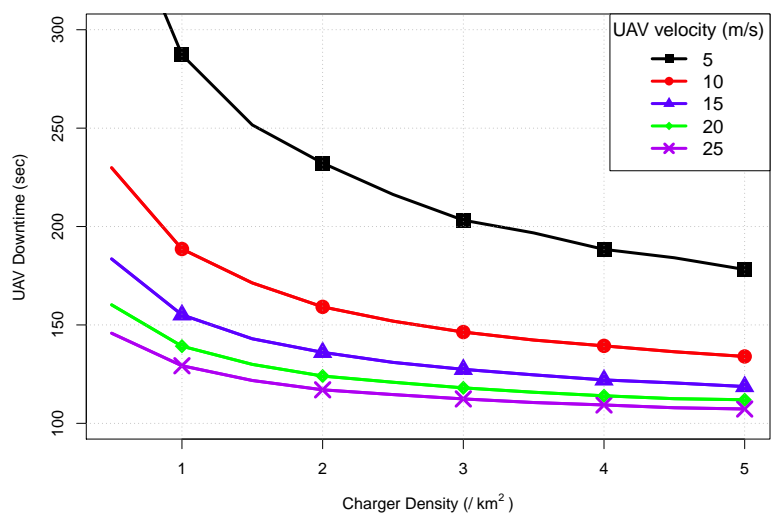

Fig. 3. Total UAV downtime under battery hotswapping, as a function of charging station density and UAV horizontal velocity.

of this downtime, as a function of horizontal UAV velocity and density of charging stations per unit area, assuming the hotswap procedure takes 60 seconds as in [7]. We can see that the total downtime will last less than 3 minutes for the majority of the UAV velocities. The 3 minutes of downtime (as per Fig. 3) may be accepable if the hotspot corresponds to regular user data traffic; for emergencies or other scenarios where the data is time-critical the operator may wish to have a backup UAV available, as in the previous section.

\section{Wireless POWER TRANSFER}

Battery hotswapping appears to be a viable solution to the limited UAV battery life. However, it still requires UAVs to regularly move between their serving location and a charging station, which reduces the operating efficiency of the network. An alternative approach is to wirelessly transfer power to the UAVs. A variety of techniques for wirelessly transferring power to a UAV have been researched. These can be roughly separated into two categories: electromagnetic field (EMF) charging and non-EMF charging. EMF charging refers to using electro-magnetic fields to transfer energy, using magnetic induction or similar. These techniques work across a very short range (in the order of centimeters) and are incapable of transferring sufficient energy quickly enough to compensate for the energy consumption of the airborne UAV. Non-EMF refers to using photo-voltaic (PV) cells to charge UAVs, using power sources such as solar radiation.

Solar power has been considered for a variety of applications, including for use in UAVs. Typically, solar-powered UAVs operate at very large altitudes in the order of tens of kilometers, as solar power generation is the most effective at larger altitudes; however, there has been some interest in applying solar power to small, low-altitude UAVs like the sort discussed in this paper. In [8] the authors present a prototype quadcopter UAV which carries a PV cell array. The authors report that the UAV is capable of staying airborne for 1-2 hours (depending on weather and payload weight), as harvesting solar power allows the UAV to offset some of the power consumed by the motors. Power consumption exceeds the power generation, so the UAV still relies on a battery supply which eventually runs out, forcing the UAV to land. As the

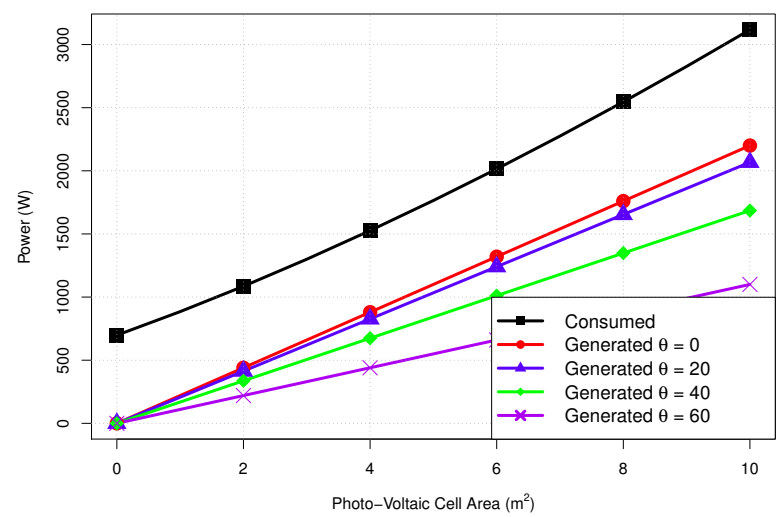

Fig. 4. Energy consumed and produced by a solar-powered UAV, as a function of the PV panel area and the solar incidence angle $\theta$.

UAV can replenish its battery supply using solar power it no longer has to land at dedicated charging stations, which gives the network more flexibility in how it operates. Note that the prototype UAV presented in [8] has a length and width of two meters; the large size may limit its ability to safely land outside of dedicated sites.

Using the UAV design parameters given in [8] we investigate the impact of the PV cell area on the UAV performance. Fig. 4 demonstrates that increasing the cell area increases the generated power, but the additional weight of the cells also increases the power consumption of the motors. We observe that the PV cells are incapable of generating sufficient power to drive the motors even when the PV cells are perfectly aligned with the sun, which means that the UAV batteries will always experience a net drain. Note that Fig. 4 presents an optimistic set of results, as we consider ideal weather conditions, while also assuming that the weight of the UAV airframe and motors does not increase with the increasing PV cell size.

As an alternative to solar power, we consider the case where the PV cells have energy beamed to them using lasers. The company Lasermotive has demonstrated a working prototype of a UAV which can remain in the air indefinitely using a kilowatt laser which transmits a beam of energy at a specially designed PV panel on the UAV [9], [10]. The difficulty with using lasers for energy transmission is that the lasers require an unobstructed LOS to the UAV to be able to reach it with their beam. In an urban environment with buildings of varying heights it may be difficult to guarantee a LOS link between a given UAV and its laser transmitter.

We explore the viability of radiative power transfer via lasers in the scenario depicted in Fig. 1(c). We assume a number of laser transmitters are mounted on rooftops in a city. A UAV deployed above a hotspot will attempt to establish a LOS link to the nearest transmitter and have the transmitter beam power to it. The expression for the energy propagation of the laser beam is given in [10], interested readers can refer to the given work for more information on laser power modelling. The beam is assumed to be deactivated if there is a LOS obstruction for safety reasons, with the LOS blockage modelled as in [5].

In Fig. 5 we plot the probability that the UAV will receive 


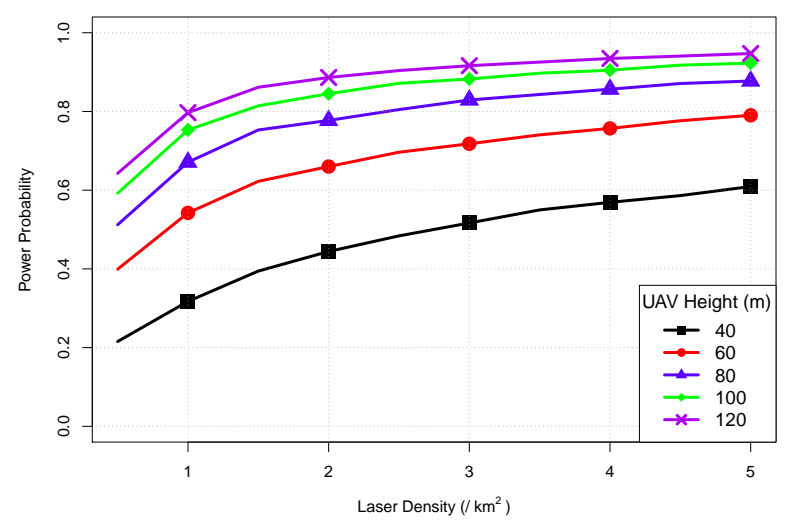

Fig. 5. Probability that a UAV hovering above a hotspot can be successfully charged by the nearest laser transmitter to it, as a function of laser density, given a laser height above ground of $30 \mathrm{~m}$

sufficient power from the laser beam to negate its power consumption (and thus remain in the air indefinitely), for varying densities of the laser transmitters. As the figure shows, the probability will depend significantly on how high the $\mathrm{UAV}$ is above ground, with greater heights making it more likely that the UAV can be wirelessly charged. The issue is that relying on wireless charging for the UAV therefore limits the heights that the UAV can operate at. Furthermore, the current legal height limit for UAVs in Europe and the USA is approximately $120 \mathrm{~m}$ which, according to our results, will not allow for guaranteed wireless charging unless the laser transmitter density is very high or the transmitters are positioned high above ground. Another issue with the laser transmitter is that it can only power a single UAV at a time, as it has to mechanically steer its laser beam towards the UAV. This limits the number of laser-powered UAVs that can be deployed in an area, as each UAV has to have its own dedicated laser transmitter when operational. Using laser transmitters to wirelessly power UAVs may be a good solution for UAVs that operate at higher altitudes than those currently envisioned by aviation authorities; however, for low-altitude UAVs operating in built-up areas it may not be the most practical solution.

\section{BATTERY ENERGy DENSITY IMPROVEMENTS}

Battery technology continues to advance at a steady pace, spurred on by the demand for greater energy density from the consumer electronics and electrical vehicle sectors. This improvement affects the cost of battery manufacture, the safety of the materials used, and the energy density of the batteries. Given that UAVs are significantly affected by their limited flight time we are particularly interested in the battery energy density, and how its improvement will improve the performance of the UAV network.

The authors of [11] suggest that historical improvement of battery energy density can be approximated as a steady $3 \%$ performance increase per year, which the authors point out is far too slow to satisfy the demands of the new, emerging technologies. Current commercially available UAVs use lithium-ion batteries with an energy density in the order of $250 \mathrm{Wh} / \mathrm{kg}$, and the research discussed in [12] suggests that

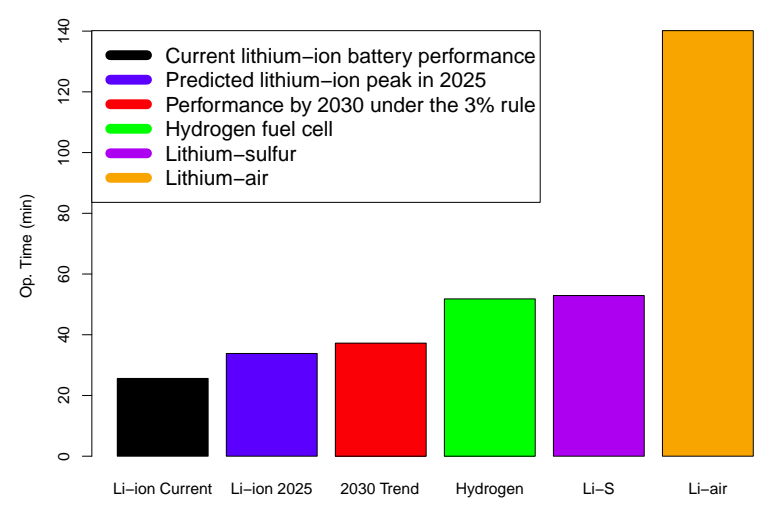

Fig. 6. Simulated UAV operating time for different battery types.

lithium-ion batteries may have their energy density improved by $20-30 \%$ within the next 5 years, reaching a performance ceiling by around 2025. So-called solid state batteries which use solid electrolytes are expected to contribute to this performance growth. Three battery technologies on the horizon that promise an improvement in energy density are the hydrogen fuel cell, the lithium-sulfur battery and the lithium-air battery, with a theoretical energy density of approximately $490 \mathrm{Wh} / \mathrm{kg}$ [13], $500 \mathrm{Wh} / \mathrm{kg}$ [14] and $1,300 \mathrm{Wh} / \mathrm{kg}$ [15], respectively. Unfortunately, these technologies have drawbacks which delay their adoption and commercialisation. There are concerns with the safety of both hydrogen fuel cells and lithium-sulfur batteries, while lithium-air batteries are known to be very vulnerable to exposure to the outside environment. Because of these drawbacks it is difficult to provide a reliable estimate on the dates when the new batteries may be adopted into UAV networks and the real-world performance these batteries will have. In Fig. 6 we aggregate the published findings to show the predicted operating time of UAVs in the coming years. A conservative estimate following the $3 \%$ annual performance increase suggests that UAVs may be able to fly in the order of 40 minutes by 2030 if hydrogen fuel, lithium-sulfur or lithiumair batteries are not commercialised by then. If they are, UAVs may be able to operate in the air for 1-2 hours at a time without needing a recharge in the not-too-distant future.

\section{CONCLUSION \& Discussion}

In this article we investigated several UAV recharging architectures that an operator may implement into a cellular network to enable the continuous use of UAVs as access points for small cells. Each of the architectures have their own strengths and weaknesses which were analysed numerically. UAV swapping can provide continuous coverage of hotspots, with the drawback of requiring backup UAVs, thus being potentially expensive. Battery hotswapping reduces the number of required UAVs, but forces the network to experience periods of downtime during which the UAVs recharge. Solar power can extend the operating time of UAVs, but currently does not appear capable of ensuring continuous flight for the multi-rotor UAVs under investigation. This may improve in time as the efficiency of PV cells and electric motors improves, and the weight of UAV materials decreases. Laser power, meanwhile, 
can allow UAVs to operate in the air as long as necessary without landing, but will experience issues with LOS blockage in the urban environment.

In addition to the strengths and weaknesses discussed in this work, there are other issues that need to be addressed before UAV charging becomes viable. For UAV swapping to be carried out seamlessly new handover protocols are needed, as entire groups of users will need to be handed over from one small cell to another without any interruption of service or handover failures. To enable battery hotswapping the UAV and charging station hardware need to be very resistant to wear-and-tear, as the mechanical manipulation of the battery by complicated actuators may be vulnerable to failure. For laser charging to be safe and reliable UAVs require very precise positioning in three-dimensional space, even in weather conditions where the UAVs will be exposed to wind. A failure to address these issues can lead to UAV network deployments which are inefficient, unreliable or unsafe.

\section{ACKNOWLEDGEMENTS}

This material is based upon works supported by the Science Foundation Ireland under Grants No. 14/US/I3110 and 13/RC/2077.

\section{REFERENCES}

[1] N. H. Motlagh, M. Bagaa, and T. Taleb, "UAV Selection for a UAVBased Integrative IoT Platform," IEEE Global Communications Conference (GLOBECOM), pp. 1-6, Dec. 2016.

[2] F. Khelifi et al., "Localization and Energy-Efficient Data Routing for Unmanned Aerial Vehicles: Fuzzy-Logic-Based Approach," IEEE Communications Magazine, vol. 56, no. 4, pp. 129-133, April 2018.

[3] S. Ahmed et al., "Energy Efficient Path Planning Techniques for UAVBased Systems With Space Discretization," IEEE Wireless Communications and Networking Conference (WCNC), pp. 1-6, April 2016.

[4] R. Gangula et al., "A Landing Spot Approach for Enhancing the Performance of UAV-Aided Wireless Networks," IEEE International Conference on Communications Workshops (ICC Workshops), pp. 1-6, May 2018.

[5] B. Galkin, J. Kibiłda, and L. A. DaSilva, "A Stochastic Model for UAV Networks Positioned Above Demand Hotspots in Urban Environments," ArXiv e-prints, Apr. 2018.

[6] S. Suman, S. Kumar, and S. De, "UAV-Assisted RF Energy Transfer," IEEE International Conference on Communications (ICC), May 2018.

[7] D. Lee, J. Zhou, and W. T. Lin, "Autonomous Battery Swapping System for Quadcopter," 2015 International Conference on Unmanned Aircraft Systems, ICUAS 2015, pp. 118-124, 2015.

[8] N. Kingry et al., "Design, Modeling and Control of a Solar-Powered Quadcopter," IEEE International Conference on Robotics and Automation (ICRA), pp. 1-8, May 2018.

[9] B. Read, "Fly By Light," Royal Aeronautical Society, Feb. 2017, accessed December 11th, 2018. [Online]. Available: https://www. aerosociety.com/news/fly-by-light/

[10] J. Ouyang et al., "Throughput Maximization for Laser-Powered UAV Wireless Communication Systems," IEEE International Conference on Communications Workshops (ICC Workshops), pp. 1-6, May 2018.

[11] C.-X. Zu and H. Li, "Thermodynamic Analysis on Energy Densities of Batteries," Energy \& Environmental Science, vol. 4, no. 8, p. 2614, 2011, accessed December 11th, 2018. [Online]. Available: http://xlink.rsc.org/?DOI=c0ee00777c

[12] T. Nozawa, "New Battery Technologies Still Years Away," Nikkei Asian Review, April 2017, accessed December 11th, 2018. [Online]. Available: https://asia.nikkei.com/Tech-Science/Tech/ New-battery-technologies-still-years-away?page $=2$

[13] J. Plaza, "Will Hydrogen Fuel Cells Help Drones Stay in the Air?" June 2017, accessed December 11th, 2018. [Online]. Available: https://www.expouav.com/news/latest/hydrogen-fuel-cells-drones/
[14] R. F. Service, "Lithium-sulfur Batteries Poised for Leap," Science, vol. 359, no. 6380, pp. 1080-1081, March 2018, accessed December 11th, 2018. [Online]. Available: http://science.sciencemag.org/content/ $359 / 6380 / 1080$

[15] M. A. Rahman, X. Wang, and C. Wen, "A Review of High Energy Density Lithium-air Battery Technology," Journal of Applied Electrochemistry, vol. 44, no. 1, pp. 5-22, Jan 2014, accessed December 11th, 2018. [Online]. Available: https://link.springer.com/ article/10.1007/s10800-013-0620-8

Boris Galkin was awarded a BAI and MAI in computer \& electronic engineering by Trinity College Dublin in 2014 and since then has been working toward the Ph.D. degree at CONNECT, Trinity College, The University of Dublin, Ireland. His research interests include small cell networks, unmanned airborne devices and cognitive radios.

Jacek Kibiłda received the M.Sc. degree from Poznań University of Technology, Poland, in 2008, and the Ph.D. degree from Trinity College, The University of Dublin, Ireland, in 2016. Currently he is a research fellow with CONNECT, Trinity College, The University of Dublin, Ireland. His research focuses on architectures and models for future mobile networks.

Luiz A. DaSilva holds the chair of Telecommunications at Trinity College Dublin, where he is the director of CONNECT, telecommunications and networks research centre funded by the Science Foundation Ireland. Prior to joining Trinity College, Prof. DaSilva was with the Bradley Department of Electrical and Computer Engineering at Virginia Tech for 16 years. Prof DaSilva is a principal investigator on research projects funded by the National Science Foundation, the Science Foundation Ireland, and the European Commission. Prof DaSilva is a Fellow of Trinity College Dublin, an IEEE Communications Society Distinguished Lecturer and a Fellow of the IEEE. 